\title{
Mobile Technologies for Promoting Health and Wellness among African American Youth
}

\author{
Donovan Hill ${ }^{1}$, Jasmine Blunt ${ }^{1}$, Terrence Pugh ${ }^{1}$, Monika Monk ${ }^{1}$, \\ Ji-Sun Kim², Woodrow W. Winchester, $\mathrm{III}^{3}$, D. Scott McCrickard ${ }^{2}$, \\ Paul Estabrooks ${ }^{4}$, and Felicia Doswell ${ }^{1}$ \\ ${ }^{1}$ Norfolk State University, Department of Computer Science, 700 Park Avenue, \\ Norfolk, VA 23504, United States \\ ${ }^{2}$ Virginia Tech, Department of Computer Science, 2202 Kraft Drive, \\ Blacksburg, VA 24060, United States \\ ${ }^{3}$ Virginia Tech, Grado Department of Industrial and Systems Engineering, 250 Durham \\ Hall, Blacksburg, VA 24061, United States \\ ${ }^{4}$ Virginia Tech, Department of Human Nutrition, Foods and Exercise, 338 Wallace Hall, \\ Blacksburg, VA 24061, United States \\ \{d.l.hill, j.s.blunt, t.l.pugh, m.p.monk\}@spartans.nsu.edu, \\ \{hideaway, wwwinche, mccricks, estabrkp\}@vt.edu, fdoswell@nsu.edu
}

\begin{abstract}
This paper describes an effort to address life-threatening diseases and health conditions through engaging use of mobile devices. The design targeted children ages 7-11, with a goal of becoming aware of the nutritional value of foods that they eat on a regular basis. The implementation efforts resulted in Health Attack, a matching and memory game that seeks to raise the knowledgelevel of participants about the foods that they eat. The evaluation of Health Attack, conducted through a demo and questionnaire administered to K-12 teachers, suggests that this type of game would be engaging for younger audiences as a first step in raising health awareness.
\end{abstract}

Keywords: mobile computing, games, games, evaluation.

\section{Introduction}

In the African-American community, there are a growing number of lifethreatening diseases that can be prevented or lessened with a healthy diet and exercise, including heart disease, cancer, and diabetes (e.g., [1,2]). We assembled a team of designers to create an engaging interface to address health and wellness concerns among African Americans. The design and implementation was led by four developers, under the guidance of two program supervisors, an academic advisor, one technical supervisor, and one health and wellness domain expert. The demographic constitution of the design team sought to ensure that people aware of issues first-hand would have a prominent role in the creation of the interfaces. It was also expected that the team would have appropriate access to ensure active end-user involvement by the target populations. To ensure appropriate leveraging of readily available 
technologies, the team selected the iPhone mobile phone as the development platform within the first week, since ownership of mobile devices is higher among African Americans and Latinos, and those groups are more likely to use their mobile device as their primary internet access device [3].

The design team sought to target a younger family demographic with their application, expecting that behavior would be easier to influence and that the lessons would be reinforced in schools and other community groups. Specifically, there was a focus on children, ages 7-11 to become nutritionally aware of the foods that they consume on a daily basis-particularly important given recent increases in childhood obesity. The paper introduces Health Attack, a matching and memory game which takes common foods found in the African American community and puts them in their respective places on the USDA food pyramid. The expectation for this application is that it will help increase the nutritional awareness of African-American children and give the children information on the food that they eat on a daily basis. The game reinforces what is learned in school about the USDA food pyramid. As it is our belief that responsiveness to cultural norms is of import in the usefulness of a health and wellness application, Health Attack leverages cultural norms and values central to the African-American community such as "role-modeling of behaviors" in convening and reinforcing nutritional messages. The Health Attack interface is described in Section 3.

Evaluation of Health Attack took place through a demo and questionnaire. The feedback questionnaire was given out to different participants, of different ethnic backgrounds, who viewed information and used this application at a highly interactive poster session. Each user was allowed to test the application; in total, sixteen users filled out the questionnaire. The evaluation is described in Section 4.

\section{Related Work}

There are a great many life-threatening diseases-many of which disproportionally affect African Americans - that can be prevented or lessened with a healthy diet and exercise, including heart disease, cancer, and diabetes (e.g., [1,2]). In particular, there have been recent increases in childhood obesity in recent years, (e.g, [4,5,6]) leading to a focus in the childhood obesity area by First Lady Michelle Obama.

Prior work suggests that mobile devices are a good match for our work. Ownership of mobile devices is higher among African Americans, and that group is more likely to use their mobile device as their primary internet access device [3]. Mobile device interfaces are showing strong potential as enablers of behavioral change, particularly in the health and wellness areas (e.g., UbiFit Garden fosters physical activity through the use of mobile displays [7], and mobile games can help African Americans make better eating and exercise decisions [8,9]).

In particular, games provide an experience for users that is both engaging and informative. Games have been leveraged previously to reach out to both adults and children, e.g., [10]. Moreover, findings suggest that computer games could be effective in promting healthier food and beverage consumption [11]; hence our research focus on developing and evaluating a mobile game for young people. 


\section{Health Attack}

A team of designers was assembled and charged with creating an engaging interface to address health and wellness concerns among African Americans. The design and implementation was led by four developers, under the guidance of two program supervisors and one technical supervisor. Five of the seven people on this team were African American, providing connections and empathy with the target population with potential for improved interfaces [12]. The team was given eight weeks to create and test their interface.

The demographic constitution of the design team sought to ensure that people aware of issues first-hand would have a prominent role in the creation of the interfaces. It was also expected that the team would have appropriate experiences and access to ensure active end-user representation by the target populations. To ensure appropriate leveraging of readily available technologies, the team selected the iPhone mobile phone as the development platform, since ownership of mobile devices is higher among African Americans and Latinos, and those groups are more likely to use their mobile device as their primary internet access device [3]. The iPhone also was the fastest growing smartphone of 2008 [13], with the largest market share of any single phone, ensuring a presence in many homes.

Many of the early group meetings focused on brainstorming ideas to meet the needs of the target population, but within two weeks the focus turned to rapid prototype development. After an initial prototype was created (by week four), the remaining time was dedicated to iteration and testing. Prototypes were presented both formally and informally to domain experts and user interface development experts to influence positively future iterations. This paper describes a summative evaluation with a group of K-12 educators (see Section 4).

The first important design decision was the application target domain. Based on the team's knowledge of the African-American community, there are a growing number of life-threatening diseases that can easily be prevented with a healthy diet and exercise-diseases such as heart disease, cancer, and diabetes that three of the main causes of deaths to African-Americans in the United States (see www.cdc.gov/omhd). These diseases have been linked to the preparation of certain foods in the African-American community known as "Soul Food"-typically, food that is prepared with heavy amounts of sodium, sugar, and fat to bring more flavoring to the food that leads to an increased risk for heart disease, high blood pressure, and high cholesterol.

The design team sought to target a younger family demographic with their application, expecting that behavior would be easier to influence and that the lessons would be reinforced in schools and other community groups. Specifically, there was a focus on children ages 7-11 to become nutritionally aware of the foods that they consume on a daily basis-particularly important given recent increases in childhood obesity. The resulting application, called "Health Attack", is a matching and memory game which takes common foods found in the African American community and puts them in their respective places on the USDA food pyramid. The expectation is to help increase the nutritional awareness of African-American children and give the children information on the food that they eat on a daily basis. Employing these lessons in a 
game created an environment targeted for children, but highly accessible by older youth and adults of all ages.

Games provide an experience for users that is both engaging and informative. Games have been leveraged previously to reach out to both adults and children, e.g., [10]. This game reinforces what is learned in school about the USDA food pyramid. As it was the developers' collective belief that responsiveness to cultural norms is of importance in the usefulness of a health and wellness application, the game leverages cultural norms and values central to the African-American community such as "rolemodeling of behaviors" in convening and reinforcing nutritional messages.

With the African American culture in mind, the developers designed the interactive game for African-American children to provide awareness of nutritional information of commonly-eaten food. Important to culturally situated design, the design team conducted a review of the literature and supported field studies while using relevant cultural elements central to the African-American community — drawing heavily from their own experiences as youth and as older siblings. An important step was to find symbols of black culture in our surrounding communities by going around the local community and to take pictures and notes about African American culture-quickly centering on issues of food selection and preparation. After finding important symbols in the community, the design team discussed how to convert that same tradition and important characteristics into the application targeted for AfricanAmerican children (though accessible to children and adults of all ages and demographics).

While preparing and reviewing candidate interface development specifications, the development team wanted to develop and choose a detailed description that could correlate well with the ideas of our findings within the African American community, listing realistic scenarios and factors that should be considered while developing the Health Attack application. The team decided to create a matching card game where players collect different food items and have them placed in their respective places on the USDA food pyramid.

While users are playing the matching game, they are also being given health facts about the food they are matching. This back and forth feedback is used to balance fun and learning, to attract users and to give them a reason to continue learning more about health and nutrition. The selection of foods that are popular in the African American community provides a sense of ownership for the target users. Also included were quotes from African Americans to serve as role models to help make the game feel more authentic and accessible to the African American community.

Health Attack was implemented using Xcode, an objective-C language intended to provide a smooth and easy workflow, with the Interface Builder toolkit to support smooth aligned images. This made development for the iPhone easy to implement in a short time frame-supporting the goals of rapid prototyping and constant user feedback. 


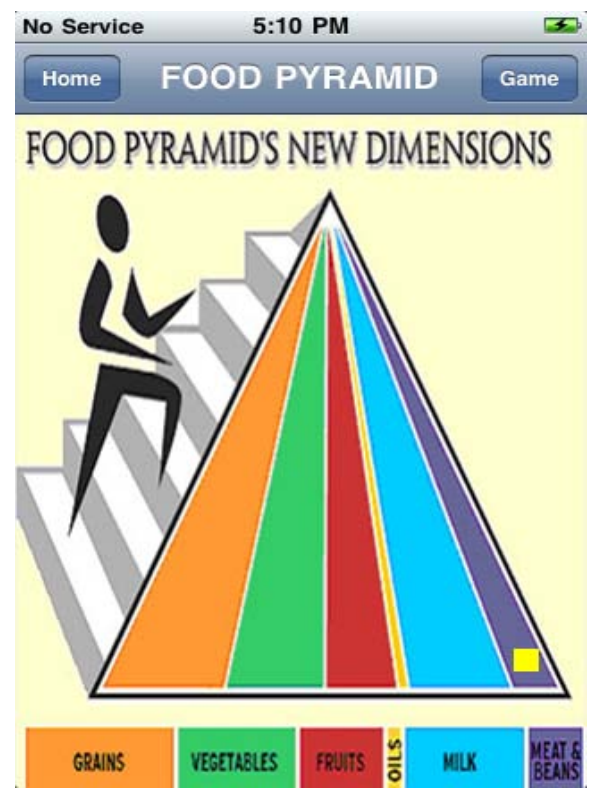

Fig. 1. Pyramid view of Health Attack, adapted from the USDA food pyramid. Each color on the pyramid corresponds to a class of foods, labeled at the bottom. Users can select any of the food types to play Health Attack with that type of food (see figure 2).

There are three major components that we implemented into this application: the USDA pyramid (figure 1), the matching game (figure 2), and the information cards (figure 3). The game alternates between these views, as described in the figures, with primary game play taking place in the matching game view. A user selects a food category in the pyramid view, taking them to the matching game (with hidden pairs of foods from the selected category).

When the user finds two matching cards, the information card view appears, with nutritional information about the food shown on the cards. The user repeats the process until all matches in the section are found, at which time the user is returned to the pyramid view. At any time during the game the user can go to the food pyramid. The user can also interact with the pyramid by clicking on the box below the sections and see the food icons of the food they have matched.

Students initial component is the USDA pyramid (figure 1). The USDA pyramid features six sections representing the different food groups: Grains, Vegetables, Fruits, Oils, Milk, and Meat \& Beans. Although there are six sections on the USDA pyramid, this application only interacts with five of them-excluding the oils section because there is little positive nutritional value about this section. Each time the user starts a new game, the USDA pyramid is empty. However when a match is made in the matching game component the USDA pyramid is updated with that matched food in its proper section; for example, the user touching or tapping a card with green beans then finding a match results in the Vegetables section in the pyramid component being automatically with green beans. The user can then click a section on the pyramid to see food icons that they previously matched. 


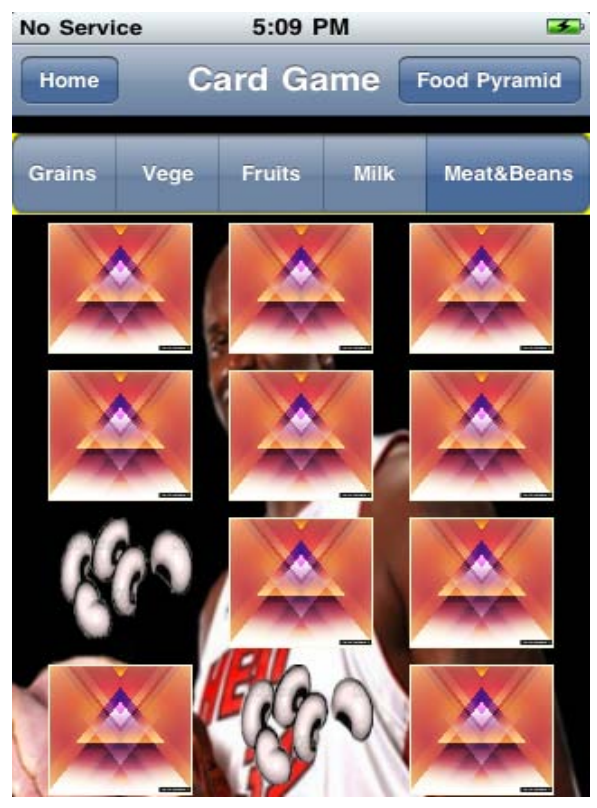

Fig. 2. Game view of Health Attack, showing a "match" of black-eyed peas selected by a user. All cards are initially face down, with a food represented on the reverse side. A user can select any two cards, and if the images match then information about the food is displayed (see figure 3).

The matching game is the main component of the application, where users spend most of their time. It consists of a $3 \times 4$ grid of cards that, when tapped, reveal cartoon images of food. It is a standard card-based matching game: the user taps or touches a card to select it, the image is revealed, then the user must select another card. If a match is made, a pop-up card with nutritional information (the information card, see figure 3) about the particular food that the user has matched comes to the front of the screen. If there is no match, both cards are hidden and the user must try again to find a match.

There are two types of information cards in this application. The first type of information cards are revealed when a match is made. These cards contain brief nutritional facts about the matched food. They also contain a picture of the food with the background color of its section in the USDA pyramid.

The second type of information cards are seen when the user enters the pyramid section and clicks on a type of food. This type of card contains two pictures of the food, as well as the background color from food's category in the USDA pyramid. This type of card also has three sections: the first is a fact section which is just a short informative fact about the food; the second section is a nutritional fact; and the third section tells the user how this type of food can be prepared (e.g., boiled, steamed, or fried). The first section gives the user something fun and informative to read. The second section gives additional detail. The final section gives the user different ways that the food can be prepared, so they can understand familiar (and healthy) options. 


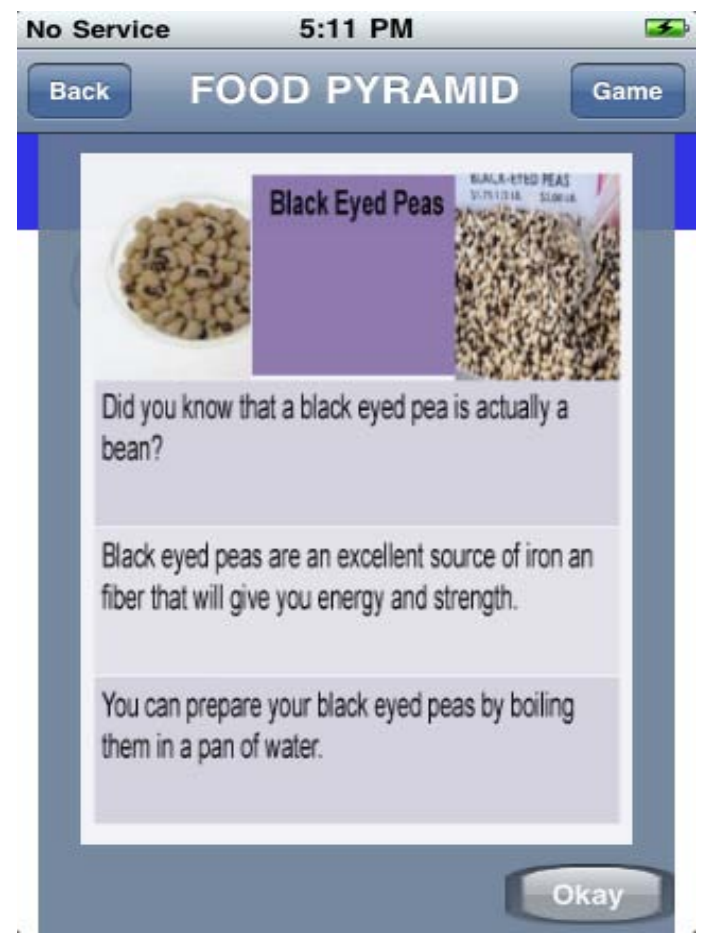

Fig. 3. Information view of Health Attack, showing data about black-eyed peas. Each card includes pictures of a food and information about it-including interesting facts, nutrition information, healthy preparation suggestions, and/or testimonials from African American role models.

Another of the cultural aspects implemented in the application are role models and foods that are authentic to the culture. The role models in the application are in the background of each of the five $3 \times 4$ grids. The role models in the backgrounds are: Barack Obama, Shaquille O'Neal, Michelle Obama, Jennifer Hudson, and an African American model. When the user has matched all of the cards in a section the background becomes visible. All of the role models have food in the picture that is related to the section they are in. Role models are a big part of any culture especially in the African American community because kids are often influenced by people they look up to and children are more likely to relate to media heroes that are of the same ethnicity [14]. The types of foods that are incorporated in "Health Attack" are foods that African Americans generally eat. Remaining authentic while not being stereotypical the application gives nutritional information about certain foods that have been considered part of the culture. 


\section{Evaluation}

Evaluation of Health Attack was conducted through a demo and questionnaire. The feedback questionnaire was given out to sixteen participants-primarily K-12 teachers, but also some college teachers and senior students. The participants viewed information on a poster, talked with developers and project leads, and experienced hands-on use of the application at a highly interactive poster session.

First, the developers discussed the background of the application. Then each participant was allowed to test the application. On the questionnaire, each participant was asked six questions. Three questions allowed the participant to rank the performance of the application on a one (worst) to ten (best) scale. The other three questions asked for each participant's free-form opinions about the application.

For the first ranking question - the overall enjoyment of the application for the participant - the average ranking for this question was 8.6, with a high of 10 and a low of 7. Based on this average and range, the participants seemed to feel as though the application provided a positive experience. The second question asked if the participant felt as though the application would be helpful to raise nutritional awareness for children, specifically African-American children; the average ranking for this question was 8.4, with a high of 10 and a low of 5. From this average, a majority of the participants felt as though this application will have a positive effect on children, specifically African American children. For the third ranking question, the participant was asked how they felt about the different role models used in the backgrounds throughout the game; the average ranking for this question was 8.5, with a high of 10 and a low of 5 . Two of the participants did not answer this question, perhaps because they did not notice the role models in the background.

On the questionnaire, the participants were also asked for their personal opinions for improvements for the game, the effectiveness of the role models used in the backgrounds throughout the game, and the relatedness of the foods to the African American culture. Many of the comments were suggestions for key components to improve the application. A couple of the participants suggested coordination with other applications — such as a food diary or log to enter in the foods they eat and get points for the foods that they eat. Other comments asked for a "search" feature for different foods. Other participants recommended that the application include more animation, sounds, and music to enhance the user experience with the application. Another recommendation from one of the participants was to add multiplayer interaction - even something as simple as a high-score log and a comments area may excite users - so that the user could be able to play and interact with other people.

When asked about the effectiveness of the role models, many of the participants responded positively to the question. One participant said that "positive association is always valuable"; another participant said "yes it raises awareness but that's the first step". However, it seems that some participants did not even notice the presence of the role models. From these positive responses, a much stronger emphasis should be placed on the role models in the backgrounds of the game.

Since many of the participants were not African American or did not understand the African American culture, they may not have felt comfortable answering the last question on the questionnaire. However, most of the participants who did respond provided positive feedback about how the food was related to African American 
culture. For the future, we wish to target audiences that are familiar with the African American culture in order to get accurate results.

\section{Conclusions and Future Work}

This paper introduced an application, Health Attack, that targets 7-11 year olds with a game-like environment for learning about healthy foods and the USDA food pyramid. We described the creation of Health Attack, including the roles of developers, interface experts, and a domain expert. An experiment with 16 participants revealed promising directions for Health Attack and similar applications.

Based on the data taken from the feedback and from the expert review of project supervisors and a domain expert, the overall perspective and effectiveness of this application is very positive. A key strength of this application is the incorporation of role models in the backgrounds throughout the game. Another strength of the game is the presence of the food pyramid in the game; many of the participants felt as though this helped with having the children learn where each food is placed.

There are several possible future directions for the game (and similar games). One is the use of sound effects and animations - an engaging way to draw users to the game. There was also concern that the language used on the pop-up messages and food pyramid information cards was too high of a level for the children to understand, requiring a match between target population and language level. Furthermore, many of the participants felt as though the information on the pyramid cards needed to be more relative to the children and also less wordy; it seems better to convey a small amount of information to all users rather than have most users ignore the information. Finally, we feel that the card game approach to conveying information is worthy of further exploration - the concise, clearly-delineated information that fits on a card provides user-digestible quantities of information that can be matched, sequenced, or otherwise associated toward furthering learning and exploration.

\section{Acknowledgements}

Thanks goes to the U.S. National Science Foundation (NSF) grants IIS-0851774 and CNS-0940358 for their support of this work. And a special thanks to the many people who used and commented on our methods and software.

\section{References}

1. Kulkarni, K. Food, culture, and diabetes in the United States. Clinical Diabetes 22 (4), 2004.

2. James, D. Factors influencing food choices, dietary intake, and nutrition-related attitudes among African Americans: Application of a culturally sensitive model. Ethnicity and Health 9 (4), 349—367, 2004. 
3. Smith, A. Mobile Access 2010. Technical report, Pew Internet \& American Life Project, 2010.

4. Kim, S. and Obarzanek, E. Childhood obesity: A new pandemic of the new millennium. Pediatrics 110 (5), 1003-1007, 2002

5. Lobstein, T., Baur, L., and Uauy, R. Obesity in children and young people: A crisis in public health. Obesity Reviews 5 (2), 4-85, 2004

6. Kumanyika, S. K., Gary, T. L., Lancaster, K. J., and Samuel-Hodge, C. D. Achieving healthy weight in African-American communities. Obesity Research 13 (2), 2037-2047, 2005

7. Consolvo, S., McDonald, D. W., and Landay, J. A. Theory-driven design strategies for technologies that support behavior change in everyday life. In: Proceedings of the ACM Conference on Human Factors in Computing Systems (CHI 2009), 405-414, 2009

8. Grimes, A. Sharing personal reflections on health locally. In: Shared Encounters: Content Sharing as Social Glue in Public Places, Springer Press.

9. Grimes, A. and Grinter, R. E. Designing persuasion: Health technology for low-income African American communities. In: Proceedings of Persuasive Technology, 24-35, 2007

10. Grimes, A., Kantroo, V., and Grinter, R. E. Let's play! Mobile health games for adults. In: Proceedings of the ACM 12th International Conference on Ubiquitous Computing (UbiComp 2010), 241-250, 2010

11. Pempek, T., and Calvert, S. Use of advergames to promote consumption of nutritious foods and beverages by low-income African American children. Archives of Pediatric Adolescent Medicine 163 (7), 633-637, 2009

12. Winchester, W. W., McCrickard, D. S., and Doswell, F. Towards culturally empowered design: Addressing African-American health disparities through notification interfaces. Workshop paper in CHI 2010 Wellness Information Workshop, 4 pages

13. Elmer-DeWitt, P. iPhone sales grew 245\% in 2008. Fortune Magazine, March 2009

14. Anderson, K. J. and Cavallaro, D. Parents or pop culture?: Children's heroes and role models. Childhood Education 78 (3), 161-169, March 2002 